http://dx.doi.org/10.1590/1678-4162-8867

Arq. Bras. Med. Vet. Zootec., v.69, n.4, p.955-961, 2017

\title{
Comparação entre vacas puras Holandês e mestiças Holandês x Jersey quanto à sanidade, imunidade e facilidade de parto
}

\author{
[Comparison between Holstein and Holstein $\times$ Jersey crossbred cows for health, \\ immunity and easy calving] \\ J.G. Dal Pizzol ${ }^{1}$, I.P.O. Gomes ${ }^{1}$, W. Braun ${ }^{1}$, J.A.N. Lisboa ${ }^{2}$, K.K.M.C. Flaiban ${ }^{2}$, A. Thaler Neto ${ }^{1}$ \\ ${ }^{1}$ Universidade do Estado de Santa Catarina - Udesc/CAV, Lages, SC \\ ${ }^{2}$ Universidade Estadual de Londrina, Londrina, PR
}

\begin{abstract}
RESUMO
O cruzamento com a raça Jersey vem sendo utilizado principalmente como alternativa para o aumento da concentração de sólidos do leite em rebanhos puros Holandês, sendo a capacidade de produção desses animais conhecida em diversos estudos. Entretanto, ainda existem limitadas informações sobre diversos fatores relevantes para a tomada de decisão e para o manejo de rebanhos cruzados, tais como resistência a doenças e facilidade de parto, sendo esta a motivação do presente trabalho, o qual objetivou avaliar a sanidade, a imunidade e a facilidade de parto de vacas mestiças Holandês x Jersey em relação a vacas puras Holandês. Foram analisados dados de dificuldade de parto, duração da gestação, retenção de placenta, indicadores sanguíneos para doenças metabólicas pós-parto (cetose e paresia puerperal hipocalcêmica) e de imunidade obtidos em vacas mestiças Holandês x Jersey e puras Holandês durante o período de um ano. $\mathrm{O}$ grupamento genético não afetou a facilidade de parto $(\mathrm{P}=0,4376)$, a retenção de placenta $(\mathrm{P}=0,7074)$ e a duração da gestação $(\mathrm{P}=0,2812)$. Vacas mestiças apresentaram maiores concentrações de gamaglobulinas $(1,776$ contra $1,456 \mathrm{~g} / \mathrm{dL})$ e de proteína total $(7,019$ contra $6,525 \mathrm{~g} / \mathrm{dL})$. Quanto à concentração de $\beta$-hidroxibutirato (BHBA), ocorreu diferença somente no dia do parto, com valores mais altos para as vacas mestiças $(0,580$ contra $0,427 \mathrm{mmol} / \mathrm{L})$. Observou-se diferença entre grupamentos genéticos para concentração de cálcio iônico $(\mathrm{P}=0,082)$, com vacas mestiças apresentando concentração mais baixa $(3,92$ contra $4,3 \mathrm{mg} / \mathrm{dL})$. Conclui-se que vacas mestiças apresentam melhor performance em indicadores de imunidade e pior nos indicadores de cetose e paresia puerperal hipocalcêmica no pós-parto em relação às puras Holandês. $\mathrm{O}$ cruzamento não aumenta o risco de distocia em vacas inseminadas com touros Holandês.
\end{abstract}

Palavras-chave: cetose, cruzamento, imunoglobulinas, paresia puerperal hipocalcêmica, retenção de placenta

\begin{abstract}
The crossbreed with the Jersey breed has been used mainly as an alternative to increasing the concentration of milk solids in Holstein herds, the production capacity of these animals having become the focus of several studies. However, there is still limited information on many relevant factors for decision-making and management of crossbreed herds, such as disease resistance and ease of calving, and this is the motivation for this work, which aimed to evaluate the health, immunity and calving difficulty in Holstein $x$ Jersey crossbred cows compared to pure Holstein cows. Data from calving difficulty, retained placenta, gestation length, blood indicators for postpartum metabolic diseases (ketosis and hypocalcemic puerperal paresis) and immunity in a herd composed by crossbreeds and Holstein cows during one year was analyzed. The genetic groups did not affect ease of calving $(P=0.4376)$, retained placenta $(P=0.7074)$ and gestation length $(P=0.2812)$. Crossbred cows had higher concentrations of gammaglobulins (1.776 versus $1.456 \mathrm{~g} / \mathrm{dL}$ ) and total protein (7.019 versus $6.525 \mathrm{~g} / \mathrm{dL})$.
\end{abstract}

Recebido em 9 de novembro de 2015

Aceito em 24 de agosto de 2016

*Autor para correspondência (corresponding author)

E-mail: andre.thaler@udesc.br 
For the concentration of BHBA, differences occurred only at calving, with higher values for crossbred cows $(0.580$ versus $0.427 \mathrm{mmol} / \mathrm{L})$. Difference was observed between genetic groups for concentration of ionized calcium $(P=0.082)$, with crossbred cows presenting lower concentrations (3.92 versus $4.3 \mathrm{mg} / \mathrm{dL}$ ). In conclusion, crossbred cows have superior performance compared to Holstein cows for immunity indicators and lower for hypocalcemic puerperal paresis and ketosis indicators on the day of calving. Crossbred cows do not have an increased risk of dystocia in relation to pure Holstein cows when mated with Holstein bulls.

Keywords: ketosis, crossbreeding, immunoglobulins, hypocalcemic puerperal paresis, retained placenta

\section{INTRODUÇÃO}

Após várias décadas de seleção para a produção, rebanhos de animais puros Holandês conseguiram aumentar significativamente a quantidade de leite produzido por animal. Em contrapartida, fatores relacionados à saúde e à fertilidade tiveram um declínio acentuado (Bjelland et al., 2011). Complicações sanitárias no período periparto, bem como complicações ocorridas durante o parto (distocias) podem gerar prejuízos para toda a lactação, como custos com medicamentos, perdas de produção e reprodutivas. Mudanças nos critérios de pagamento do leite também estão ocorrendo no Brasil, de modo que aspectos como o conteúdo de sólidos do leite e a contagem de células somáticas passaram a ser mais valorizados ao estabelecerem as estratégias de melhoramento genético dos rebanhos leiteiros.

Alterar uma característica dentro de uma raça normalmente é um processo lento devido às baixas herdabilidades em muitas características de interesse econômico, podendo demorar várias gerações para se obterem mudanças significativas. Com isso, o uso do cruzamento, principalmente com a raça Jersey, surge como uma alternativa mais rápida para a solução de problemas relacionados à melhoria na composição de sólidos no leite e na fertilidade, entre outros.

Vacas mestiças Holandês x Jersey se mostraram superiores quanto ao percentual de sólidos do leite em relação às puras Holandês, em trabalhos como os de Heins et al. (2008) e Thaler Neto et al. (2013), e também quanto à eficiência reprodutiva, longevidade e facilidade de parto (Heins et al., 2008; Brown et al., 2012; Pinedo et al., 2014). Porém, quanto à produção de leite, vacas mestiças Holandês x Jersey apresentam uma pequena diferença; vacas $1 / 2$ Holandês $\mathrm{x}$ Jersey produzem o equivalente a $92-94 \%$ do volume de leite produzido pelas vacas puras Holandês (Heins et al., 2008; Thaler Neto et al., 2013). Aspectos sanitários e peculiaridades do período de transição pós-parto em vacas mestiças Holandês $\mathrm{x}$ Jersey ainda não foram bem avaliados.

Apesar de suas vantagens quanto à composição de leite e de índices reprodutivos, a raça Jersey apresenta maior predisposição genética a algumas doenças, como paresia puerperal hipocalcêmica (Lean et al., 2006). Aliado a isso, devido ao menor porte dos animais frutos do cruzamento entre as raças Holandês e Jersey, quando estes foram comparados às vacas puras Holandês, gerou-se uma preocupação quanto à facilidade de parto e ao possível aumento na prevalência de doenças do período periparto. Com isso, o objetivo do presente trabalho foi comparar a sanidade no período de transição pósparto, a imunidade e a facilidade de parto de vacas puras Holandês e mestiças Holandês x Jersey.

\section{MATERIAL E MÉTODOS}

O estudo foi realizado em uma propriedade localizada no município de Carambeí - PR (245' $21.16^{\prime \prime}$ Sul e $50^{\circ} 05^{\prime} 11,21^{\prime \prime}$ Oeste e 1038 metros de altitude), a qual possui animais puros Holandês e mestiços Holandês x Jersey de diversas frações genéticas Os animais foram divididos em dois grupamentos genéticos, sendo vacas puras Holandês $(\mathrm{n}=29)$ e mestiças Holandês $\times$ Jersey $(n=26)$, com frações $1 / 2$ Holandês x $1 / 2$ Jersey, $3 / 4$ Holandês x $1 / 4$ Jersey e $5 / 8$ Holandês x $3 / 8$ Jersey.

As vacas permaneciam em pastagem com suplementação de volumoso e concentrado o ano todo, sendo separadas em dois lotes, conforme a produção de leite. O lote de maior produção (média de $40 \mathrm{~kg} / \mathrm{dia}$ ), com consumo de matéria seca estimado em $23,6 \mathrm{~kg}$ vaca/dia, 
recebia aproximadamente $14 \mathrm{~kg}$ de alimentos concentrados, e o lote de menor produção (média de $25 \mathrm{~kg} / \mathrm{dia}$ ), com consumo estimado em $20,4 \mathrm{~kg}$ vaca/dia, recebia aproximadamente $7,9 \mathrm{~kg}$ provenientes de alimentos concentrados. As vacas eram secas 60 dias antes da previsão de parto, sendo alimentadas com pastagem e cerca de $2 \mathrm{~kg} / \mathrm{dia}$ de concentrado. A ordenha era realizada duas vezes ao dia.

Durante o período de um ano, foram avaliados indicadores reprodutivos (facilidade de parto, retenção de placenta, duração da gestação), ocorrência de doenças metabólicas subclínicas (paresia puerperal hipocalcêmica e cetose) e imunidade (proteínas totais do soro sanguíneo e imunoglobulinas no pós-parto). Todas as vacas foram inseminadas com touros Holandês, com valores genéticos para facilidade de parto nos Estados Unidos variando de 6 a 9\% de partos difíceis.

Dados de facilidade de parto das vacas foram anotados seguindo uma escala de um a cinco, sendo 1 para parto fácil, sem auxílio, e 5 para extrema dificuldade, cesariana ou morte, conforme proposto por Chung et al. (2008). Para fins de análise, os partos foram classificados em atócicos (valores de 1 a 3 ) e distócicos (valores 4 e 5). Foi considerada retenção de placenta quando, após 24 horas do parto, não houve o desprendimento dos envoltórios fetais, conforme Walsh et al. (2007).

Foi avaliada a concentração de $\beta$-hidroxibutirato no sangue nas primeiras 24 horas após o parto e na nona semana pós-parto, como indicador de ocorrência de cetose subclínica, utilizando-se um equipamento eletrônico portátil (Option Xceed ${ }$, Abott do Brasil), conforme indicado por Voyvoda e Erdogan (2010).

As concentrações de cálcio total e iônico no soro sanguíneo de vacas nas primeiras 24 horas após o parto foram avaliadas como indicadores de ocorrência de paresia puerperal hipocalcêmica subclínica. O soro foi obtido após a coagulação do sangue coletado dos vasos coccígeos, separado e congelado a $-20^{\circ} \mathrm{C}$. A dosagem do cálcio total foi realizada pelo método colorimétrico cresolftaleína, no Laboratório de Análises Clínicas da Universidade do Estado de Santa Catarina Udesc, Campus de Lages. As concentrações de cálcio iônico (CaI) foram estimadas pela concentração do cálcio total, da albumina e da proteína total no soro sanguíneo.

A imunidade pós-parto foi analisada por meio da dosagem de gamaglobulinas no soro sanguíneo obtido nas primeiras 24 horas após o parto. As concentrações de proteínas totais e de gamaglobulinas foram determinadas no Laboratório de Patologia Clínica do Hospital Veterinário da Universidade Estadual de Londrina - PR (UEL). As proteínas totais foram analisadas em espectrofotômetro semiautomático, com filtro de $545 \mathrm{~nm}$, pelo método de biureto. As concentrações séricas de gamaglobulinas foram determinadas por eletroforese em gel de agarose (Kremers et al., 1967), (Sistema SE-250, CELM $®)$. A proporção de cada fração proteica foi estabelecida por meio do programa computacional SDS-60 do sistema SE-250 (CELM $®)$.

As análises estatísticas foram realizadas utilizando-se o pacote estatístico SAS (SAS Institute, 2002). Os dados binários de facilidade de parto e retenção de placenta foram analisados por meio do emprego de um modelo linear generalizado, com distribuição binomial (regressão logística), utilizando-se o procedimento GENMOD. Para facilidade de parto, o modelo estatístico foi constituído pelas variáveis grupo genético (Holandês e mestiças Holandês x Jersey), paridade (primípara e multípara) e sexo da cria. Para retenção de placenta, utilizou-se um modelo estatístico similar, substituindo-se o efeito da variável sexo da cria pelo efeito da facilidade do parto (eutocia ou distocia).

Os dados das concentrações de $\beta$-hidroxibutirato no sangue, cálcio total, cálcio iônico, IgG, proteína total no soro sanguíneo e duração da gestação foram submetidos à análise de variância, utilizando-se o procedimento GLM, sendo previamente testados para normalidade dos resíduos pelo teste de Shapiro-Wilk. O modelo estatístico foi constituído pelas variáveis grupo genético (Holandês e mestiças Holandês $\mathrm{x}$ Jersey), paridade (1, 2 e $\geq 3$ ) e estação de parto (inverno, primavera, verão e outono). Para a variável duração da gestação, foi incluída a variável sexo da cria no modelo.

O projeto foi aprovado pelo Comitê de Ética em Experimentação Animal da Universidade do 
Estado de Santa Catarina (Cetea/Udesc), protocolo 01.11.10.

\section{RESULTADOS E DISCUSSÃO}

Não foi observada diferença entre os grupamentos genéticos quanto à duração da gestação (Tab. 1). Normam et al. (2009) avaliaram aproximadamente 11 milhões de partos em sete rebanhos na Flórida e encontraram valores semelhantes para duração da gestação em vacas das raças Jersey e Holandês (279,4 e 280,0, respectivamente), enquanto Silva et al. (1992) observaram gestação mais curta em vacas da raça Jersey comparadas a vacas puras Holandês (278 contra 280 dias). Dhakal et al. (2013) também observaram duração da gestação semelhante ao compararem vacas puras Holandês (274,2 $\pm 0,7$ dias), mestiças Holandês x Jersey $(273,6 \pm 0,7$ dias) e Jersey (273,6 $\pm 0,8$ dias), quando inseminadas com touro Holandês.
Foi observado efeito de ordem de parto sobre a duração da gestação $(\mathrm{P}=0.0148)$. As novilhas apresentaram gestação mais curta quando comparadas às vacas a partir da segunda parição (276,89 contra 279,87 dias). Diversos estudos demonstram gestações mais curtas em novilhas, como o de Norman et al. (2009).

Também não foi observada diferença na facilidade de parto entre fêmeas puras e mestiças inseminadas com touros Holandês (Tab. 1). Nos Estados Unidos, Weigel e Barlass (2003) observaram que, na opinião dos produtores, as diferenças em dificuldade de parto também eram pequenas, com leve vantagem para as vacas $\mathrm{F} 1$ Holandês x Jersey em relação às puras Holandês, quando ambas eram inseminadas com touros Holandês. Dhakal et al. (2013) também não observaram diferença na dificuldade de parto ao compararem vacas Holandês, mestiças Holandês $\mathrm{x}$ Jersey e Jersey, quando inseminadas com touros Holandês.

Tabela 1. Duração da gestação, facilidade de parto e retenção de placenta em vacas puras Holandês e mestiças Holandês x Jersey

\begin{tabular}{ccccccc}
\hline \multirow{2}{*}{$\begin{array}{c}\text { Grupamento } \\
\text { genético }\end{array}$} & \multicolumn{2}{c}{ Duração da gestação } & \multicolumn{2}{c}{ Facilidade de partos } & \multicolumn{2}{c}{ Retenção de placenta } \\
\cline { 2 - 7 } & $\mathrm{N}$ & dias $\pm \mathrm{EPM}^{*}$ & $\mathrm{~N}$ & \% partos difíceis & $\mathrm{N}$ & \% casos retenção \\
\hline Holandês & 26 & $279,11 \pm 0,82$ & 27 & 14,81 & 21 & 14,29 \\
Mestiços & 22 & $277,78 \pm 0,89$ & 22 & 9,09 & 28 & 10,71 \\
\hline & & $\mathrm{P}=0,2812$ & $\mathrm{P}=0,7813$ & & $\mathrm{P}=0,7074$
\end{tabular}

* Erro-padrão da média.

Foi observada retenção de placenta em 12,24\% das vacas, não havendo influência do grupamento genético (Tab. 1). O resultado está de acordo com o trabalho de Anderson et al. (2007), ao avaliarem vacas puras Holandês comparadas com um grupamento formado por vacas Jersey e mestiças Holandês x Jersey em confinamento nos Estados Unidos, os quais encontraram $3,7 \%$ e $4,3 \%$ de retenção de placenta, respectivamente, sendo, entretanto, estes valores inferiores aos do presente estudo. Por outro lado, prevalências de retenção de placenta semelhantes ao do presente trabalho têm sido citadas em outros trabalhos, como o de Corassin et al. (2011), ao avaliarem vacas puras Holandês de alta produção no Brasil, os quais observaram 19\% de retenção de placenta.

Em relação à imunidade, as vacas mestiças apresentaram maiores concentrações de gamaglobulinas e proteína total no soro sanguíneo no dia do parto em comparação às vacas puras Holandês (Tab. 2). Estes resultados aproximam-se dos encontrados por Feitosa e Birgel (2000), em vacas puras Holandês (1,98 e $6,81 \mathrm{~g} / \mathrm{dL}$ ) para gamaglobulinas e proteína total no soro sanguíneo no momento do parto. Maior concentração de imunoglobulinas no momento do parto pode propiciar redução na ocorrência de doenças infectocontagiosas. Dal Pizzol et al. (2014), em um estudo com o mesmo rebanho do presente trabalho, observaram menor contagem de células somáticas nas vacas mestiças Holandês x Jersey em relação às puras Holandês $(\mathrm{P}<0,0001)$. Em uma pesquisa realizada no estado de Santa Catarina, Felippe (2013) também observou redução na ocorrência de infecções uterinas puerperais $(\mathrm{P}=0,0125)$, com menor prevalência nos grupamentos com maior percentual de Jersey na composição genética. 
Comparação entre vacas...

Tabela 2. Valores de gamaglobulinas e proteína total no soro de vacas puras Holandês e mestiças Holandês x Jersey no dia do parto

\begin{tabular}{lccc} 
& $\mathrm{N}$ & Gamaglobulinas g/dL & Prot. total g/dL \\
\hline Holandês & 23 & $1,456 \pm 0,1065$ & $6,525 \pm 0,1493$ \\
Mestiças & 26 & $1,776 \pm 0,1002$ & $7,019 \pm 0,1404$ \\
\hline & $\mathrm{P}=0,0389$ & $\mathrm{P}=0,0233$ \\
\hline
\end{tabular}

Foi observado efeito de grupamento genético sobre a concentração de $\beta$-hidroxibutirato (BHBA) no dia do parto. As vacas mestiças apresentaram valores mais elevados em relação às puras Holandês (Tab. 3), não havendo, entretanto, diferença significativa nos níveis de
BHBA na nona semana pós-parto. Segundo Wittwer (2000), os valores aceitáveis de BHBA são de $0,5 \mathrm{mmol} / \mathrm{L}$, salvo vacas no início de lactação, quando podem ser considerados normais valores de até $0,8 \mathrm{mmol} / \mathrm{L}$.

Tabela 3. Média de BHBA no dia do parto e na nona semana de lactação de vacas puras Holandês e mestiças Holandês x Jersey

\begin{tabular}{lcccc}
\hline & Dia do parto & & $9^{\text {a }}$ semana \\
\hline & $\mathrm{N}$ & BHBA mmol/L & $\mathrm{N}$ & BHBA mmol/L \\
Holandês & 29 & $0,4027 \pm 0,0615$ & 11 & $0,2247 \pm 0,0233$ \\
Mestiças & 19 & $0,5802 \pm 0,0498$ & 16 & $0,2001 \pm 0,0193$ \\
\hline & $\mathrm{P}=0,0304$ & $\mathrm{P}=0,4482$ \\
\hline
\end{tabular}

Anderson et al. (2007), quando compararam vacas Holandês com vacas Jersey e mestiças Holandês x Jersey, encontraram maior incidência de cetose no grupamento Jersey e mestiças em relação ao grupamento Holandês (12,3 contra $5,1 \%$, respectivamente). Em pesquisas realizadas em um rebanho experimental nos Estados Unidos, não foram observadas diferenças entre vacas Holandês, Jersey e mestiças F1 dessas raças quanto à prevalência de cetose clínica (Olson et al., 2011), assim como para a concentração de ácidos graxos não esterificados ao longo da lactação (Brown et al., 2012), o que está de acordo com os resultados observados no presente experimento quanto à concentração de BHBA na nona semana de lactação.

Foi observado efeito de ordem de parto sobre a concentração de $\beta$-hidroxibutirato no soro sanguíneo $(\mathrm{P}=0,0018)$, aumentando sua concentração com o aumento da ordem de parto. Brown et al. (2012) também observaram maior concentração de ácidos graxos não esterificados em vacas de segunda lactação em relação às primíparas.

Não foi observada diferença entre os grupamentos genéticos quanto ao nível de cálcio total no soro sanguíneo no dia do parto $(\mathrm{P}=$ $0,1163)$, havendo efeito mais pronunciado sobre a concentração de cálcio iônico $(\mathrm{P}=0,0802)$, sendo os valores encontrados para vacas mestiças inferiores aos das vacas puras Holandês (Tab. 4). Concentrações mais baixas de cálcio total e iônico podem ser esperadas em vacas mestiças, devido às baixas concentrações desse mineral no soro sanguíneo de vacas Jersey no dia do parto, demonstrado em diversos trabalhos. Curtis et al. (1984) relacionam o fato ao menor número de receptores para 1,25-di-hidroxivitamina D (DHVD) no intestino delgado nas vacas Jersey. Ballantine e Herbein (1991), ao compararem vacas Holandês e Jersey, observaram menores concentrações de cálcio total no plasma sanguíneo de vacas Jersey no dia do parto $(7,47$ contra $8,10 \mathrm{mg} / \mathrm{dL}$ ), assim como de cálcio iônico $(4,25$ contra $4,66 \mathrm{mg} / \mathrm{dL})$.

Os resultados observados indicam que rebanhos de animais mestiços Holandês x Jersey precisam de atenção especial quanto ao manejo periparto, devido ao risco mais elevado do aparecimento de quadros clínico e subclínico de paresia puerperal hipocalcêmica. Os valores médios de cálcio total, para ambos os grupamentos genéticos (Tab. 4), estão abaixo da concentração normal de cálcio no plasma sanguíneo, a qual, segundo Andrews et al. (2008), varia de de 8,8 a $10,4 \mathrm{mg} / \mathrm{dL}$ e, segundo Goff et al. (1991), de 8,0 a 10,0 mg/dL. 
Tabela 4. Concentração de cálcio total e iônico no soro sanguíneo de vacas puras Holandês e mestiças Holandês x Jersey e em diferentes ordens de parto

\begin{tabular}{ccc} 
& Cálcio total $(\mathrm{mg} / \mathrm{dL})$ & Cálcio iônico $(\mathrm{mg} / \mathrm{dL})$ \\
\hline Grupo genético & & \\
Holandês & $7,84 \pm 0,058$ & $4,30 \pm 0,0318$ \\
Mestiços & $7,20 \pm 0,052$ & $3,92 \pm 0,0281$ \\
\hline & $\mathrm{P}=0,1163$ & $\mathrm{P}=0,0802$ \\
\hline Ordem de parto & \\
1 & $8,581 \pm 0,3244$ & $4,647 \pm 0,1777$ \\
2 & $7,093 \pm 0,3244$ & $3,849 \pm 0,1777$ \\
$\geq 3$ & $6,999 \pm 0,3453$ & $3,827 \pm 0,1891$ \\
\hline
\end{tabular}

Foi observado efeito de ordem de parto sobre as concentrações de cálcio total $(\mathrm{P}=0,002)$ e iônico $(\mathrm{P}=0,035)$, sem haver interação com $\mathrm{o}$ grupamento genético. Chan et al. (2006) também observaram efeito de ordem de parto sobre a concentração de cálcio total no soro sanguíneo de vacas puras Holandês, e verificaram que vacas primíparas obtiveram maiores concentrações de cálcio em comparação às vacas multíparas. Segundo DeGaris e Lean (2009), o risco de uma vaca adoecer de hipocalcemia aumenta cerca de $8 \%$ a cada lactação, relacionado à diminuição da capacidade de mobilização de Ca dos ossos, e, de acordo com Van Mosel et al. (1993), há diminuição da absorção do cálcio no intestino, devido à diminuição dos receptores de DHVD.

\section{CONCLUSÕES}

Vacas mestiças apresentam melhor performance em indicadores de imunidade e pior nos indicadores de cetose e paresia puerperal hipocalcêmica no pós-parto em relação às puras Holandês. O cruzamento não aumenta o risco de distocia em vacas inseminadas com touros Holandês.

\section{AGRADECIMENTOS}

À Granja RAG, nas pessoas dos senhores Raul e Ricardo Guimarães e família, por disponibilizar os dados e os animais, assim como pela grande colaboração para a realização deste estudo. À CAPES pelo apoio financeiro e pela concessão de bolsa de mestrado ao primeiro autor.

\section{REFERÊNCIAS}

ANDERSON, T.; SHAVER, R.; BOSMA, P.; DE BOER, V. Case study: performance of lactating Jersey and Jersey-holstein Crossbred versus Holstein cows in a Wisconsin confinement dairy herd. Prof. Anim. Sci., v.23, p.541-545, 2007.

ANDREWS, A.H.; BLOWEY, R.W.; BOYD, H.; EDDY, R.G. Medicina bovina: doenças e criação de bovinos. São Paulo: Roca, 2008. 1067p.

BALLANTINE, H.T.; HERBEIN, J.H. Potentiometric determination of ionized and total calcium in blood plasma of Holstein and Jersey cows. J. Dairy Sci., v.74, p.446-449, 1991.

BJELLAND D.W., WEIGEL K.A., HOFFMAN P.C. et al. Production, reproduction, health, and growth traits in backcross Holstein $\times$ Jersey cows and their Holstein contemporaries1. J. Dairy Sci., v.94, p.51945203, 2011 .

BROWN, K.L.; CASSELL, B.G.; MCGILLIARD, M.L. et al. Hormones, metabolites, and reproduction in Holsteins, Jerseys, and their crosses. J. Dairy Sci., v.95, p.698-707, 2012.

CHAN, P.S.; WEST, J.W.; BERNARD, J.K. Effect of prepartum dietary calcium on intake and serum and urinary mineral concentrations of cows. J. Dairy Sci., v.89, p.704-713, 2006.

CHUNG, Y.H.; PICKETT, M.M.; CASSIDY, T.W.; VARGA, G.A. Effects of prepartum dietary carbohydrate source and monensin periparturient metabolism and lactation in multiparous cows. $J$. Dairy Sci., v.91, p.2744-2758, 2008.

CORASSIN, C.H.; MACHADO, P.F.; COLDEBELLA, A. et al. Importância das desordens do periparto e seus fatores de risco sobre a produção de leite de vacas Holandesas. Semin. Ciênc. Agrár., v.32, p.1101-1010, 2011. 


\section{Comparação entre vacas...}

CURTIS, C.; ERB, H.; SNIFFEN, H.; SMITH, R. Epidemiology of parturient paresis: predisponing factors with emphasis on dry cows feeding and management. J. Dairy Sci., v.64, p.817-825, 1984.

DAL PIZZOL, J.G.; THALER NETO, A.; FARIAS, D.K. et al. Contagem de células somáticas em vacas da raça holandesa e mestiças Holandês x Jersey. Arch. Vet. Sci., v.19, p.46-50, 2014.

DEGARIS, P.J.; LEAN, I.J. Milk fever in dairy cows: a review of pathophysiology and control principles. Vet. J., v.176, p.58-69, 2009.

DHAKAL, K.; MALTECCA, C.; CASSADY, J.P. et al. Calf birth weight, gestation length, calving ease, and neonatal calf mortality in Holstein, Jersey, and crossbred cows in a pasture system. J. Dairy Sci., v.96, p.690-698, 2013.

FEITOSA, F.L.F.; BIRGEL, E.H. Variação da concentração de imunoglobulinas $\mathrm{G}$ e $\mathrm{M}$, de proteína total e suas frações eletroforéticas e da atividade da gamaglutamiltransferase no soro sangüíneo de vacas holandesas, antes e após o parto. Arq. Bras. Med. Vet. Zootec., v.52, p.11-116, 2000.

FELIPPE, E.W. Comparação de vacas mestiças das raças Holandesa x Jersey com vacas puras quanto à eficiência produtiva e reprodutiva. 2013. 54f. Dissertação (Mestrado em Ciência Animal) Programa de Pós-Graduação em Ciência Animal, Universidade do Estado de Santa Catarina, SC.

GOFF, J.P.; REINHARDT, T.A.; HORST, R.L. Enzymes and factors controlling vitamin D metabolism and action in normal and milk fever cows. J. Dairy Sci., v.74, p.4022-4032, 1991.

HEINS, B.J.; HANSEN, L.B.; SEYKORA, A.J. et al. Crossbreds of Jersey $x$ Holstein compared with pure Holsteins for production, fertility, and body and udder measurements during first lactation. J. Dairy Sci., v.91, p.1270-1278, 2008.

KREMERS, B.; BRIERE, R.O.; BATSAKIS, J.G. Reflectance densitometry of cellulose acetate protein electrophoresis. Am. J. Med. Technol., v.33, p.28-34, 1967.

LEAN, I.J.; DEGARIS, P.J.; MCNEIL, D.M.; BLOCK, E. Hypocalcemia in dairy cows: metaanalysis and dietary cation anion difference theory revisited. J. Dairy Sci., v.89, p.669-684, 2006.
NORMAN, H.D.; WRIGHT, J.R.; KUHN, M.T. et al. Genetic and environmental factors that affect gestation length in dairy cattle. J. Dairy Sci., v.92, p.2259-2269, 2009.

OLSON, K.M.; CASSELL, B.G.; HANIGAN, M.D.; R.E., PEARSON. Interaction of energy balance, feed efficiency, early lactation health events, and fertility in first-lactation Holstein, Jersey, and reciprocal F1 crossbred cows. J. Dairy Sci., v.94, p.507-511, 2011.

SILVA, H.M.; WILCOX, C.J.; THATCHER, W.W. et al. Factors affecting days open, gestation length, and calving interval in Florida dairy cattle. J. Dairy Sci., v.75, p.288-293, 1992.

STATISTICAL analysis system. Cary: SAS Institute, 2002.v.1, 349p.

THALER NETO, A.; RODRIGUES, R.S.; CÓRDOVA, H.A. Desempenho produtivo de vacas mestiças Holandês x Jersey em comparação ao Holandês. Rev. Ciênc. Agrovet., v.12, p.7-12, 2013.

VAN MOSEL, M.; VAN, T.; KLOOSTER, A.T. et al. Effects of reducing dietary $[(\mathrm{Na}++\mathrm{K}+)-(\mathrm{CI}-+$ $\mathrm{SO} 4=) 1$ on the rate of calcium mobilisation by dairy cows at parturition. Res. Vet. Sci., v.54, p.1-9, 1993.

VOYVODA, H.; ERDOGAN, H. Use of a hand-held meter for detecting subclinical ketosis in dairy cows. Res. Vet. Sci., v.89, p.344-351, 2010.

WALSH, R.B.; WALTON, J.S.; KELTON, D.F. et al. The effect of subclinical ketosis in early lactation on reproductive performance of postpartum dairy cows. $J$. Dairy Sci., v.90, p.2788-2796, 2007.

WEIGEL, K.A.; BARLASS, K.A. Results of a producer survey regarding crossbreeding on US dairy farms. J. Dairy Sci., v.86, p.4148-4154, 2003.

WITTWER, F. Diagnóstico dos desequilíbrios metabólicos de energia em rebanhos bovinos. In: GONZÁLEZ, F.H.D.; BARCELLOS, J.O.; PATINO, H.O.; RIBEIRO, L.A. Perfil metabólico em ruminantes: seu uso em nutrição e doenças nutricionais. Porto Alegre: Universidade Federal do Rio Grande do Sul, 2000. p.9-22. 